\title{
The new genomic medicine service and implications for patients 胱结
}

\author{
Authors: Katie Snape, ${ }^{A}$ Sarah Wedderburn ${ }^{B}$ and Julian Barwell ${ }^{C}$
}

In January 2019, a new nationally commissioned Genomic Medicine Service (GMS) has now commenced in the NHS. Capitalising on the infrastructure developed through the 100,000 Genomes Project, the GMS is underpinned by seven supra-regional Genomic Laboratory Hubs (GLHs) delivering the new inherited rare disease and cancer somatic tissue genetic test directory. This replaces the UKGTN test directory, with the aim of standardising criteria for whole genome sequencing or targeted panel tests where applicable. The new test directory will define who can order specific genetic tests under prescribed eligibility criteria. In keeping with Dame Sally Davies' white paper Generation Genome, this will further democratise genetic testing and, in some situations, avoid the need to refer to clinical genetics to access testing. The aim is to simplify patient pathways and reduce regional or social inequalities. We will discuss the implications of whole genome sequencing and the potential impact of the new nationally commissioned GMS for both patients, their relatives and clinicians. We will also discuss the imminent challenges in implementing genomic medicine into the NHS, and the future impact of novel technologies on service delivery as genomic medicine becomes increasingly integrated into routine healthcare.

KEYWORDS: Genomic Medicine Service, devolved nations

\section{Introduction}

Over the past decade, transformative advances in DNA sequencing technologies have enabled a vast expansion in analysis of the human genome for the purpose of diagnosing and managing human disease. The scope of genetic testing now ranges from analysing a single gene, larger panels of multiple genes and the coding region of the genome (the exome) through to the whole 3 billion base sequence of the human genome.

An exemplar of the impact this can have on patient management is in the field of oncology. By undertaking whole genome sequencing of paired DNA extracted from both a

Authors: ${ }^{\text {A }}$ consultant clinical geneticist and honorary senior lecturer, St George's University of London, London, UK; ${ }^{\text {s }}$ specialty registrar in clinical genetics, NHS Greater Glasgow and Clyde, Glasgow, UK; ' Consultant clinical geneticist, Leicester Royal Infirmary, Leicester, UK patient's blood lymphocytes (constitutional genetic testing) and cancer tissue (somatic genetic testing), the clinician can identify the underlying molecular processes driving the disease to assist with therapeutic decisions, as well as identifying any potential underlying genetic predisposition to cancer, which might influence future disease risk or an increased risk for relatives.

Thus far in the UK, large scale genomic analyses have primarily taken place through the 100,000 Genomes Project. ${ }^{1}$ A new nationally commissioned Genomic Medicine Service (GMS) has now commenced in the NHS. Capitalising on the infrastructure developed through the 100,000 Genomes Project, the GMS is underpinned by seven supra-regional Genomic Laboratory Hubs (GLHs) delivering the new inherited rare disease and cancer somatic tissue genetic test directory. ${ }^{2}$ The test directory specifies eligibility criteria for testing, and indicates who can order the test. An increasing number of genetic tests can be ordered in primary and secondary care, often referred to as 'mainstreaming'. For example, an endocrinologist can now order a gene panel test for patients they suspect to have familial hypocalciuric hypercalcaemia $(\mathrm{FHH})$, and a neurologist can order a gene panel test for early onset dementia without first having to refer to a clinical geneticist, assuming they meet the test criteria set out in the National Test Directory. ${ }^{2}$

Whole genome sequencing (with specific gene panel analysis) will be a first line test for patients in specific circumstances such as some cancer, metabolic, neurological and ophthalmological disorders. However, the vast majority of both somatic and constitutional genetic testing will be undertaken using smaller gene panels. These gene panels will include known diagnostic, prognostic and therapeutic targets. These seismic changes in the way genetic testing is undertaken will impact across medical specialties, and require clinical evolution of the roles of both mainstream healthcare workers and clinical geneticists and genetic counsellors (GCs).

In this overview, we propose recommendations to help facilitate the integration of genomic data into routine medical care.

\section{The medical specialty: clinical genetics interface}

The reconfiguration in genetic testing will initiate innovation in the ways that medical specialties and clinical genetics services interact with each other. The role of the clinical geneticist or GC has traditionally been to identify individuals who have an increased susceptibility to developing a given disease due to variation within their genetic code, and assist with the interpretation of genomic data to enable the treating physician to make therapeutic and management decisions. 
The type of genomic data being received by mainstream specialties will increase and diversify rapidly over the next few years. In the near future, medical clinicians are likely to find themselves interpreting pharmacogenomic data to enable more personalised prescribing, genomic data on pathogens to guide antibiotic decisions and track outbreaks, complex genomic data underpinning multifactorial disease to guide screening and for risk stratification, alongside testing for traditional high risk genes causing Mendelian disease.

This will involve clinical transformation at many levels, particularly educational initiatives, and increased interaction between the physicians receiving genomic data and the genetic scientists and clinicians who can assist in accurate interpretation and translation (Table 1).

$>$ Recommendation. Develop education and governance teams both centrally and at local trust level to ensure smooth transition to mainstreaming of diagnostic testing with appropriate and timely referrals to clinical genetics. Resource clinical genetic services to provide top down education and training to medical specialties.

The treating physician can now directly request and receive genetic information, without a referral, assessment and analysis by clinical genetics services (Table 2 ). The cardiologist, suspecting

Table 1. Educational and support requirements for medical students and doctors

\section{Grade and position}

Medical student middle grade clinicians

Consultants and associate specialists

Foundation years and core medical trainees

\section{Required transformation}

Updated and taught undergraduate genomics curriculum reflecting transformation in quantity and diverse clinical application of genomic data.

Updated junior doctor training curricula. 'On the job' training in pharmacogenomics and prescribing. Clear pathways for requesting advice from clinical genetics services.

Specific genomics curriculum and integrated training as relevant to specialty. For example, oncology trainees receiving cancer genetics training attachments in the identification, investigation and management of patients at increased risk of genetic predisposition to cancer. Clear pathways for requesting advice from clinical genetics services.

Reactive training programmes in genomic data and clinical applications. Novel, efficient pathways for receiving specialist genomic advice from both clinical genetics services and genomic laboratory hubs. Implementation of new testing and management pathways due to mainstreamed genetic testing.
Table 2. Genomic Medicine Service and impact on

genetic testing and consent
Scenario

Medical diagnosis is clear and there is a suspected genetic aetiology seen by a medical specialist (in some complex dysmorphology settings, clinical genetics will still see the patient first)

Patient with cancer history but no mutation known to be present

Known mutation in the family

\section{Plan for the Genomic Medicine} Service

Mainstreamed constitutiona genetic testing by medical specialty with referral to clinical genetics if a family history is also present or a significant variant identified.

Reflex somatic (tumour) testing for treatment with referral to clinical genetics if a family history is also present or a somatic variant identified which could indicate underlying inherited cancer predisposition gene. Mainstreamed constitutional genetic testing by cancer team (oncology/surgery) with referral to clinical genetics if a family history is also present or a significant variant identified.

Seen by clinical genetics with increasing use of risk modelling involving clinical and genomic data.

Direct referral to clinical genetics for predictive testing.
Marfan syndrome in his tall patient with aortic root dilatation, can consent the patient for genetic testing, arrange for a blood test for an aortopathy gene panel and receive back the report without any input from clinical genetics services. This would streamline the diagnostic pathway, but also requires the cardiologist to be confident in taking informed consent, interpretation of genetic results and to make necessary onwards referrals for patients with a confirmed genetic diagnosis.

The clinical genetics service will assist with complex interpretation of genomic data, complex or intractable cases, assessment of individuals identified to have a variant causing genetic disease, risk assessment of complex disease and predictive testing where a pathogenic variant has been identified in a family.

\footnotetext{
$>$ Recommendation. Develop implementation teams that can support consent and data entry during transition to full mainstreaming and implementation of the test directory.
}

\section{Service implementation challenges, future proofing and potential solutions}

Practical aspects of sample collection, registration, tracking and result delivery

Pathways for sample collection and delivery of genetic results are not yet embedded into medical pathways. At the time of writing, practical logistics around sample collection, data storage, 
registration and tracking of samples for patients have yet to be finalised. There is a hope that recent transformative projects such as fresh tumour testing without the use of formalin that damages DNA and mainstreaming of genetic testing in some areas of secondary care through the 100,000 Genomes Project will act as a catalyst to introducing wider molecular tests routinely into clinical practice.

The delivery of samples for the 100,000 Genomes Project has often involved dedicated teams assisting with study governance, eligibility queries, patient registration, consent, sample processing and assisting clinicians with phenotyping entry into dedicated software. If this is going to be replaced by a generic system without funding for capital investment or support staff, considerable care will be required in making this intuitive as many secondary care teams will not have dedicated additional time to learn and use the systems and may only need to use it sporadically. Although education will be important, consistently receiving results that alter and improve patient management will be key to convincing colleagues of its importance.

The National Genomic Information System (NGIS) will provide a national framework for patient registration, consent, phenotypic data entry, sample bar code labelling and tracking, and results. This will also assist with referrals into clinical genetics but has yet to be fully developed or tested.

> Recommendation. Invest in local infrastructure to embed pathways developed through the 100,000 Genomes Project. Ensure diagnostic and clinical commissioning are integrated so that diagnostic pathways have maximum clinical impact. Implement the NGIS in a safe and efficient way.

\section{Consent and genetic counselling}

Genetic counselling for patients and their families to enable informed consent to be taken for genetic testing is evolving. How, where and by whom patients are consented for genetic testing is changing. A universal 'patient choice' form will be available online and all clinicians undertaking testing will be expected to complete this. The cardiologist consenting the patient for the aortopathy panel, and the endocrinologist consenting the patient for the $\mathrm{FHH}$ panel will both need to fill out the relevant online information alongside the patient.

Embedding genomics into routine care could be the catalyst for healthcare professionals to become part of the wider genetics team. However, this will be a considerable challenge given the current time pressures and demands on professionals. Robust consent pathways need to be drawn up and disseminated with clear guidance on who should and can take consent, and educational initiatives to ensure clinicians taking consent feel equipped to do so. Easy access to GCs and clinical geneticists in complex cases will be required. A workforce shortage already looms in genetic counselling and will need to be addressed by increased training of individuals confident in taking consent and able to train others.

In cancer patients, the requirement to take consent for somatic (tumour) testing $+/$ - constitutional genetic testing alongside consent for a generic surgical or biopsy creates time and educational challenges for the clinician. A patient may find themselves launched into a situation where a genetic change which causes inherited cancer predisposition is identified through a test they believed was undertaken for their cancer management,

\section{Box 1. The exemplar of cancer}

Increased tumour testing to guide acute clinical management of the cancer patient is happening apace, and is embedded in the new test directories. Currently, there is no immediate plan to implement paired constitutional sequencing for cancer patients undergoing somatic (tumour) genetic testing unless the first line test is whole genome sequencing. Therefore, a direct clinical interface between the oncologist and geneticist is required to enable the interpretation of somatic data with respect not only to acute cancer management, but also to embed referral and management pathways into inherited cancer services where it is possible that a heritable susceptibility to cancer may also be present. Most cancer predisposition genes increase the risk of multiple tumours. It is important that the possibility of additional primary tumours is discussed with the patient and that at-risk relatives requiring cancer prevention, screening advice or cascade genetic testing can be identified.

One possible way of developing these new pathways is through increasing engagement of cancer geneticists at both central molecular tumour boards and local oncology multidisciplinary team (MDT) meetings. However, the significantly fewer numbers of cancer geneticists vs numbers of oncology MDTs presents a logistic challenge to this model. Training in cancer genomics is embedded in the medical oncology training curriculum and this must take the form of practical experience, with training oncologists spending time with clinical geneticists and training in the interpretation of somatic genomics data identified in tumour tissue. Innovations, such as using digital family history assessment to identify those at increased multifactorial risk, alongside new pathways for referral into clinical genetics services on the basis of somatic test findings are required. Both centralised and local guidelines and pathways need to be implemented to enable integrated use of molecular data for the management of both the acute cancer and relapse, but also prediction of future cancer risk and risk to relatives. This will embed both precision medicine and Screening, Prevention and Early Detection (SPED) measures into routine care pathways.

and they are not ready to deal with the consequences for them or their relatives, particularly where there is uncertainty or complex family dynamics. Cancer clinicians and genetic clinicians are aware of these issues and currently working to develop an infrastructure to embed these new testing and consent pathways (see Box 1).

$>$ Recommendation. Educate, train, explain and simplify consent pathways so that these are accessible to both mainstream clinicians and genetic clinicians. Involve patients and public in consent pathways.

\section{Data interpretation}

The breadth of genomic sequencing can lead to a complicated set of downstream consequences such as identifying variants of uncertain significance (VOUS) and secondary findings that need to be considered at the time of consent. ${ }^{4}$ There is approximately a $1-3 \%$ chance of identifying an incidental variant - one which has consequences for patient health but is not related to the diagnostic reason the test was initially undertaken, for example identifying a mutation in a cancer predisposition gene in a patient being investigated for early onset diabetes. 
We all have several hundred potentially protein altering variants that can be difficult to interpret.

The cardiologist undertaking the aortopathy gene panel is hoping to confirm a diagnosis of Marfan syndrome by finding a clearly disease causing variant in the FBN1 gene. What happens if a rare variant of uncertain significance in the ACTA2 gene is instead identified? What will the cardiologist tell the patient? Will they be comfortable interpreting this and making a clinical management decision on the basis of this?

The American College of Medical Genetics and Genomics have provided guidelines and recommendations for both variant interpretation and management of incidental findings, and the consent process for testing has had to become even more robust. ${ }^{5,6}$ Data has overtaken current knowledge and so resolving pathogenicity of a VOUS is often not readily available. Translating this to patients is often unsatisfactory. Greater investment is needed to be able to interpret these results from a laboratory perspective, alongside national and international databases, and greater investment is also needed for counselling of these results, particularly for mainstream clinicians who are not adequately trained in the interpretation of such variants but may well be delivering the results to patients.

> Recommendation. Improved, clear genetic test reports, and clear lines of communication between laboratory scientists, mainstream clinicians and clinical geneticists will hopefully enable the most effective and accurate interpretation of such results, with the most evidence-based clinical translation. This will be supported by the use of specialist laboratories that can assist with assessing the pathogenicity of unclear variants. Genomic input at specialty multidisciplinary teams may become the norm, but training of more clinicians who are able to interpret genomic data in the clinical context is urgently required.

\section{Big data and use of technology}

'Quantity' is becoming an increasing issue for the effective implementation of genomic data into improved clinical care for patients and their relatives. Constitutional whole genome sequencing requires significant data storage and analysis. Widening access, novel eligibility pathways and improved equity of access to genetic testing will increase the volume of genomic data alongside increasing the numbers of patients requiring further assessment through inherited disease services.

The government have highlighted genomics as an area ripe for digital revolution in the recent Topol Review call for evidence. ${ }^{8}$ It is easy to see how artificial intelligence (AI), in which machines can perform tasks requiring intellectual processing, could enable the expansion of genomics. It is impossible to imagine how the genomics revolution can be clinically implemented without a future in which we use technology to acquire data inputs required for risk analysis (eg family history, clinical and molecular phenotype), alongside assimilation, analysis and interpretation of genomic, molecular, clinical and radiological information from large data sets to enable accurate risk prediction. In theory, this will enable the clinician to focus more on the clinical skills requiring human interaction - face-to-face shared decision making, with a holistic approach encompassing educational, cultural and personal issues. In the future, AI may also encroach on these traditional areas as more emotional and psychosocial technological tools develop. ${ }^{9}$ The experienced clinician will need to be at the vanguard of developing and innovating using the novel tools available to them. The technology requires sensible, unbiased and clinically evidenced implementation to be most effective.

Genomics will increasingly be used for population health, as well as in specialised groups of patients. The future potential of genomics in healthcare can be illustrated with population-based screening for complex disorders, such as cancer or diabetes, defining to whom it is offered based on risk and not age alone. Being able to reduce morbidity and mortality by identifying individuals at risk for developing a condition is not a new concept but screening at a genomic level is. It is unlikely that the NHS will be in a position to roll out population based genomic screening imminently due to a lack of evidence about the potential harms and benefits of embarking on genomic screening programmes and concerns regarding affordability and workforce requirements. ${ }^{10}$ However, it should be recognised that this could be a direction that population screening takes for many conditions in future.

$>$ Recommendation. Invest in bioinformatic and digital infrastructures and resources, such as clinical software developers to enable digital transformation. Support and resource start up initiatives, and develop ways to rapidly share clinically excellent innovation nationally. Development of an ethical and technical cyber security team aiming to test strength and improve data protection systems. Develop links with machine-based learning teams and ensure entry of clinical outcome data to establish opportunities for better prediction modelling in the future. Undertake research into evidencebased use of genomic data for public health purposes to guide wider implementation.

\section{Impact on devolved nations}

England is poised for its seven Genomics Central Laboratory Hubs, but where does that leave the devolved nations? Each has a different commissioning pathway: National Services Division (NSD) for Scotland, Regional Medical Services Consortium for Northern Ireland and the Health Commission Wales for Wales. Scotland, for example, has developed the Scottish Genetic Laboratory Consortium and the Molecular Pathology Consortium to act as single designated multi-site national specialist services. Four regional centres work together to maximise efficiency and minimise duplication of work. ${ }^{11}$ Each centre is responsible for expertise in different areas. It remains unclear how the devolved nations will interact with England but it is unlikely these will be delivered in isolation given requirements for data sharing, training and similarities in service delivery.

\section{Conclusions}

We believe that challenges exist which need to be addressed to enable physicians to make increasing use of genomic data in patient care. These include the clinician being unaware of or unable/unwilling to order genetic tests due to training or time constraints; unavailability of the testing pathway locally; a lack of bioinformatic capacity or staff and the referring clinician being unable to interpret the significance of a genetic report in the context of the wider phenotype or family history with respect to variant interpretation or limitations of the test.

These implementation and educational challenges are not insubstantial and considerable investment will be required to 
deliver this at scale and pace. Our recommendations are not exhaustive but may reduce the clinical risk and maximise the clinical benefit of routine integration of genomic data into mainstream healthcare.

The implementation of the national GMS holds great promise for the improved diagnosis and management of patients and their relatives. However, there are many logistical challenges to ensuring that genomic data is effectively and efficiently integrated into existing clinical pathways. Forward thinking about workforce planning, issues around consent and data protection and harnessing the power of technological advances will be essential to maximise the benefit of the genomics revolution for healthcare purposes.

\section{Conflicts of interest}

Dr Julian Barwell has previously received conference talk presentation fees from AstraZeneca.

\section{References}

1 Turnbull C, Scott RH, Thomas E et al. The 100,000 Genomes Project: bringing whole genome sequencing to the NHS. BMJ 2018;361:k1687.

2 NHS England. National Genomic Test Directory. NHS England. www.england.nhs.uk/publication/national-genomic-test-directories [Accessed 08 October 2018]

3 Davies SC. Annual report of the Chief Medical Officer 2016: Generation genome. London: DoH, 2017.

4 Roche MI, Berg JS. Incidental findings with genomic testing: Implications for genetic counseling practice. Curr Genet Med Rep 2015:3:166-76.
5 Richards S, Aziz N, Bale S et al. Standards and guidelines for the interpretation of sequence variants: a joint consensus recommendation of the American College of Medical Genetics and Genomics and the Association for Molecular Pathology. Genet Med 2015;17:405-24.

6 Green RC, Berg JS, Grody WW. ACMG recommendations for reporting of incidental findings in clinical exome and genome sequencing. Genet Med 2013;15:565-74.

7 Weitzel JN, Blazer KR, MacDonald DJ, Culver JO, Offit K. Genetics, genomics and cancer risk assessment: State of the art and future directions in the era of personalized medicine. CA Cancer ] Clin 2011;61:327-59.

8 Health Education England. The Topol Review: Preparing the healthcare workforce to deliver the digital future. Interim report. London: HEE, 2018.

9 Wolfe CR, Reyna VF, Widmer CL et al. Efficacy of a web-based intelligent tutoring system for communicating genetic risk of breast cancer: a fuzzy-trace theory approach. Med Decis Making 2015:35:46-59.

10 Prince AER, Cadigan RJ, Henderson GE et al. Is there evidence that we should screen the general population for Lynch syndrome with genetic testing? A systematic review. Pharmgenomics Pers Med 2017:10:49-60.

11 NHS National Services Scotland. National Services Division. NHS www.nsd.scot.nhs.uk.

Address for correspondence: Dr Julian Barwell, consultant/ honorary professor in clinical genetics and genomic medicine, Leicester Royal Infirmary, Leicester LE1 5WW, UK.

Email: julian.barwell@uhl-tr.nhs.uk 